\title{
Zur Abgrenzung der sog. Alzheimerschen Krankheit.
}

\author{
Von \\ Dr. J. G. Schnitzler, \\ Assistent der neurologischen Klinik (Prof. Heilbronner) Utrecht. \\ Mit 1 Textfigur und 2 TafeIn. \\ (Eingegangen am 17. Juli 1911.)
}

Das Studium der mit schwereren organischen Hirnveränderungen einhergehenden - von Kraepelin neuerdings unter dem Namen „Alzheimersche Krankheit“" in der neuesten Auflage seines Lehrbuches zusammengefaßten - präsenilen und senilen psychotischen Zustände hat uns zweifellos die Aussicht eröffnet, aus der schwierig zu verstehenden Psychopathologie des verfrühten und ,,normalen“ Seniums eine Gruppe abzuspalten, die hinsichtlich ihrer klinischen Symptomatologie in Übereinstimmung mit im gewissen Maße spezifisch-anatomischen Veränderungen eine nosologische Sonderstellung zwingend beansprucht. Der Versuch der detaillierten Abgrenzung ist dagegen, wie man ruhig behaupten kann, aus der spärlichen Kasuistik, die bis jetzt vorliegt, wohl nur ohne Aussicht auf Erfolg möglich und führt tatsächlich zu einer allerdiffusesten Qualifizierung des Krankheitsbegriffes sowohl in klinischer wie - wenn auch scheinbar in geringerem Grade - in anatomischer Hinsicht. Als Kraepelin seinerzeit die Zustände wenigstens anatomisch fundieren zu können meinte, mit den Alzheimerschen scharf charakterisierten Fibrillenveränderungen und den senilen Plaques (,Drusen“), wobei er ausdrücklich hervorhob, daß ,,die klinische Deutung dieser Alzheimerschen Krankheit zurzeit noch unklar ist", war die Frage des Krankheitsbegriffes sogar einfacher als jetzt, nachdem Alzheimer selbst einen offenbar in diese Gruppe gehörigen Fall veröffentlicht hat, bei dem die spezifischen Fibrillenveränderungen, die doch a priori den Plaques gegenüber das wichtigere und wesentliche anatomische Substrat darstellen dürften, fehlen. Andererseits stellt sich übrigens, soweit man den Schwerpunkt des spezifischen Befundes möglicherweise doch noch in dem Vorkommen der drusigen Veränderungen erblicken möchte, hier dieselbe Schwierigkeit entgegen: es sind jetzt von Alzheimer auch Fälle veröffentlicht worden obne Drusen; und bei diesen fakultativen Verhältnissen zweier in rein histologischer Beziehung völlig ungleichwertiger und vielleicht ganz unabhängiger anatomisch-patho- 
gnomischer Befunde steht also eine sich zwingend aufdrängende, einheitliche Würdigung der dem Krankheitsbilde zugrundeliegenden anatomischen Prinzipien vorderhand wieder aus.

Von den auch bei anderen Zuständen beschriebenen gliösen und Markfaser-Veränderungen und weiteren, nicht als spezifisch für die Krankheit geltenden Befunden können wir hierbei vorläufig ganz Abstand nehmen.

Klinisch ist von einer Fixierung überhaupt keine Rede: die verschiedenartigsten psychotischen Erscheinungen ohne reines Bild einer klassischen Psychose, eventl. kompliziert durch psychische Ausfallserscheinungen von herdförmigem Charakter und Andeutungen von somatischen Herderscheinungen bilden in Zusammenhang mit dem sehr weitgefaßten - präsenilen Alter des Kranken das Wesentliche, was bis jetzt von den verschiedenen Autoren beobachtet worden ist.

Die bessere Auskrystallisierung des Krankheitsbildes fordert also voraussichtlich noch ein großes kasuistisches Material, und wenn auch ein einzelner Fall eo ipso nur einen bescheidenen Wert in dieser Hinsicht besitzt, so scheint es mir doch von Interesse, die Krankheitsbefunde einer in unserer Klinik verpflegten Patientin mitzuteilen, um so mehr, wo dieser Fall zweifellos geeignet ist, wenigstens einige neue Gesichtspunkte zu bringen.

Der freundlichen Aufforderung Herrn Prof. Alzheimers, dem ich die mikroskopischen Präparate vorlegte, und der den Fall als Beitrag zu der Frage für außerordentlich wichtig hielt, komme ich hiermit gerne nach.

Die Krankengeschichte ist die folgende:

Am 10. Dez. 1908 besucht die Kranke, Frau van D., 34 Jahre alt, zum ersten Male unsere Poliklinik.

Die aus den Angaben des Arztes und des Ehemannes kombinierte Anamnese lautet:

Vorher stets gesund gewesen. Seit 9 Jahren verheiratet. In der ersten Zeit nach der Verheiratung war sie, wie der Ehemann versichert, vielleicht nicht ganz so gescheit wie der Durchschnitt, ,etwas dumm“, doch übrigens eine ordentliche Hausfrau, die ihren Pflichten in der Haushaltung und in der Schenkwirtschaft ihres Mannes gewissenhaft und sorgfältig nachkam. Alkohol gebrauchte sie nicht. Von den 4 Kindern sind 2 gesund, 2 gestorben im Alter von 3 Monaten resp. 3 Tagen. Kein Abortus; das letzte Kind prämatur (7 Monate). Für Lues keine Anhaltspunkte. Menses regelmäßig.

Nach dem letzten Partus fiel ihrem Manne auf, das Pat. stiller wurde, ohne Beschäftigung öfter auf einem Stuhl saß, viel schlief, auffallend leicht sich über den Verlust des Kindes hinwegsetzte, kurz, im allgemeinen einen trägen und schläfrigen Eindruck machte. Die Anfangszeit dieses Zustandes datiert ungefähr 2-3 Jahre zurück. Diese Indolenz ward langsam schlimmer, außerdem nahm die Kranke auffällig in Körpergewicht zu, ,ward dick im Gesicht und am Körper". Im weiteren Verlaufe der Krankheit zeigte Pat. immer weniger Interesse für ihre Umgebung, war ,faul“" und konnte ,den ganzen Tag schlafen“, redete sehr wenig, 
benahm sich kindisch, indem sie über alles lachte, vernachlässigte ihre Kinder, gab diesen in allem nach. Essen konnte sie immer gut. In der Führung des Haush altes ward sie allmählich ganz unbrauchbar; zuerst konnte sie einfache Speisen noch kochen, schließlich ward auch dies unmöglich; sie ließ entweder den Ofen ausgehen, oder die Speisen anbrennen, oder verga $\beta$ Wasser beizugießen usw.

Zuletzt betrug sie sich ,wie ein Kind", sah wohl, nachher auf ihre Fehler aufmerksam gemacht, oft ein, was sie verkehrt gemacht hatte.

Nie Erregungszustände oder ängstliche Phasen. Aufnahme in die Klinik. Status praesens (vgl. Fig. 1): Pat. ist auffallend dick; namentlich ihr

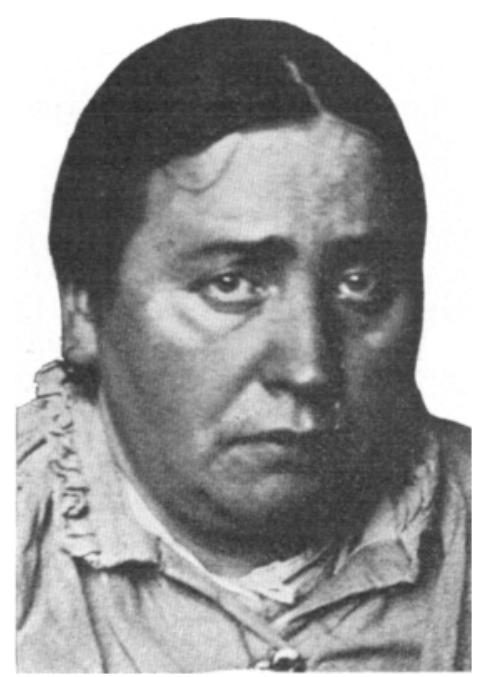

Fig.1. Gesicht sieht gedunsen aus; die Gesichtshaut ist beim Palpieren etwas myxödematös, an der Stirn sind feine Runzeln nahezu fixiert. Der Körper ist plump, verunstaltet durch schwere Hautfalten, Arme und Beine sind fast formlose Massen. Überall ist die Haut verdickt, Fingereindrücke bleiben einige Zeit stehen; die Hände sind am wenigsten geschwollen und an den Endphalangen scheinbar etwas zugespitzt. Die Hautfarbe ist nicht blaß, sondern gleichmäßig rosafarben. Der Haarwuchs ist nicht stark; die Nägel bieten nichts Abnormales, viele cariöse Zähne. Körpergewicht $100 \mathrm{~kg}$.

Die Kranke spricht langsam, dann und wann etwas schneller; man bekommt den Eindruck, daß sie zum Antworten eine ziemlich lange Reaktionszeit braucht, wenn sie einmal im Gange ist, geht es etwas flotter. Auch Bewegungen werden ziemlich akkurat ausgeführt, es dauert aber längere Zeit, bevor sie überhaupt einer Aufforderung dazu nachkommt.

Eine kleine, etwas harte Glandula thyreoidea wird - auch chirurgischerseits palpiert. Das Herz ist etwas vergrößert; im Urin kein Eiweiß oder Zucker.

Temperatur normal. Puls 90-100.

Die Kranke fällt den übrigen Kranken als abnormal auf, ist fortwährend schläfrig. Der R. Hallux steht fast permanent in „Friedreichscher" Stellung. Keine weitere körperliche Symptome.

Augenhintergrund normal.

Pat. lacht kindisch um alles, was vorfällt. Will nach Haus, ist schon einige Male aus dem Saale gelaufen, will ihre Angehörigen besuchen (!), ist damit einverstanden, wenn man ihr zuredet, daß das nicht geht, geht doch jedesmal wieder nach der Tür. Bereitet übrigens dem Pflegepersonal keine Schwierigkeiten. Reagiert im allgemeinen träge. Nur das Davonlaufen macht sie aus eigener Initiative.

\section{Dezember 1908.}

Rechnen:

$5 \times 6=30$ in 2 Sekunden.

$15 \times 3=49,45$ in 7 Sekunden.

$12 \times 7=48$ in 6 Sekunden.

$14 \times 3=42$ in $211 / 5$ Sekunden.

$17 \times 4=68$ in $24^{4} / 5$ Sekunden (rechnet $4 \times 10=40,4 \times 7=28,68$ ).

$12 \times 8=96$ in $43 / 5$ Sekunden. 
$18 \times 3=54$ in $9^{3} / 5$ Sekunden.

$13 \times 8=42$ in $9^{3} / 5$ Sekunden.

$16 \times 7=$ rechnet $7 \times 10=70,7 \times 6=42$, zählt nicht zusammen.

$21 \times 11=$ nach 12 Minuten: „Kann ich nicht so schnell ausrechnen."

Zählt von 1-50 richtig in 27 Sekunden.

Von 50-1 läßt sie 22 aus, zählt in 62 Sekunden.

Die Monate nennt sie fehlerlos in $5^{3} / 5$ Sekunden, zählt von $1-20$ in $5^{1 / 5}$ Sekunden, von 20-1 fehlerlos in 10 Sekunden.

Untersuchung an Heilbronnerschen Bildchen:

Lam pe.

1. Weiß nicht.

2. Weiß nicht.

3. Lampe, es ist immer dasselbe.

4. Auch eine Lampe.

Kirche.

1. Kirche mit Turm.

2. [Was hinzugekommen?] Zeigt annähernd + .

3. [Was hinzugekommen?] Zeigt + .

4. [Was hinzugekommen?] Zeigt + .

5. [Was hinzugekommen?] Zeigt + . Sagt: Die Häuschen drin, die Dinger (sind die Fenster).

6. [Was hinzugekommen?] Zeigt + .

7. [Was hinzugekommen?] Zeigt + .

8. [Was hinzugekommen ?] Ist dasselbe.

Tanne.

1. Tanne.

2. [Was hinzugekommen ?] Weiß nicht.

3. [Was hinzugekommen?] Zeigt + .

Kanone.

1. Weiß nicht.

2. Dasselbe mit einem Stern drin.

3. Dasselbe, weiß nicht was es ist; Unterschied + .

Schubkarren.

1. Weiß nicht.

2. Weiß nicht; Unterschied + .

3. Schubkarren.

4. Unterschied + .

5. Unterschied + .

Schiff.

1. Weiß nicht.

2. Weiß nicht; Unterschied + .

3. Weiß nicht; Unterschied + .

4. Weiß nicht; Unterschied + .

5. Schiff.

Pat. gibt sehr schnell Antwort, die Untersuchung - die, nebenbei bemerkt, von anderen, nicht defekten, Kranken ebenso wie jede detallierte Aphasieuntersuchung oft als ,,kindisch“ abgewiesen wird, oder wenigstens Schwierigkeiten in dieser Hinsicht bereitet - interessiert sie offenbar sehr; fortwährend will sie selbst schon die nächstfolgenden Zeichnungen sehen und die Blätter wenden.

Gezeigt werden ihr Wettervorhersagungen, aus der Zeitung (,Der Telegraph“) ausgeschnitten, in der Form der Darstellung eines Mannes mit einem Regen 
schirm oder einer Dame mit Sonnenschirm usw. Darüber gedruckt: Der Telegraph verkündet für heute:

Pat. sieht die Darstellungen: „Ein Herr mit einem Regenschirm“, sagt sie, indem sie diese Zeichnung betrachtet, liest das Gedruckte erst mit einer verbal paraphasischen Wendung, dann richtig. Die Bildchen sind alle gleich; ,von einem Abreißkalender", fügt sie spontan hinzu, ,stammen sie nicht.“

[Kennen sie den Telegraph?] ,Ja, es gibt eine Zeitung, die Telegraph heißt und es gibt einen Telegraph, wo man telegraphieren kann."

„Ja, die Bildchen sind aus der Zeitung.“

[Was stellt es nun dar?] Liest wieder das Gedruckte, ,das steht auf allen in gleicher Weise drauf.“

[Was bedeutet Regen?] „Der Mann mit dem Schirm.“

Liest dann eine Annonce aus der Zeitung ohne Fehler.

Der Gesamteindruck ist, daß neben der Hemmung ein Defektzustand besteht, die Demenz ist aber offenbar nicht so erheblich, daß hiermit das Gesamtbild ihres Zustandes ohne weiteres eine Erklärung finden dürfte.

Die Bourdonsche Probe, das Unterstreichen bestimmter Buchstaben in einer Leseprobe, macht sie im allgemeinen mit ziemlich viel Auslassungen, streckenweise aber fehlerlos.

Am 18. Dezember 1908 wird die Kranke von einem unserer Assistenten in der chirurgischen Klinik dem Medizinischen Verein vorgestellt; lief hier aus dem Warteraum weg; die Schwester mußte ihr bis ans andere Ende des Korridors nachlaufen, sie wollte wieder zu einem Angehörigen auf Besuch. Auf einen Stuhl gesetzt, schläft sie öfters während der Demonstration ein; ihre Bewegungen sind stark verlangsamt.

19. Dezember 1908. Weiß am Tage nachher, daß sie in einem großen Krankenhaus war; es machte fast den Eindruck eines Theaters, obwohl es keins war; viele Herren saßen da, ihr ward sogar etwas bang. Erinnert sich, daß sie auf einem Stuhl saß und daß der Assistent da war. Weiß nicht, ob dieser stand oder saß. Weiß, daß einer der Herren gesprochen hat, meint (unrichtig!), daß der Professor es wäre; der Herr sagte, daß sie so dick wäre geworden in den letzten Jahren und daß sie 45 Jahre alt wäre (richtig!). Weiß, daß sie das korrigiert hat. Bleibt dabei, daß der Professor gesprochen hat.

[Wer hat Ihnen die Bildchen gezeigt ?] Weiß es nicht.

[Was ist Ihnen gezeigt?] Weiß es nicht.

[Haben Sie in ein Buch gesehen?] Nein.

[Ein kleines schwarzes Buch?] Nein.

[Mit Zeichnungen?] $\mathrm{O}$ ja.

[Welche Bilder waren es?] Weiß ich nicht mehr.

[Tiere oder so etwas?] Ein Schubkarren.

[Auch Häuschen ?] Ja.

[Welche Häuschen ?] Ein Haus mit einem Turm.

28. Dezember 1908. Pat. hat seit der Aufnahme 2,2 kg abgenommen. Bekommt seit 10 Tagen Trochisci Gland. Thyreoid. (Burroughs Wellcome), 1 Stück pro die.

Sie weiß nicht, daß sie in den ersten Tagen nach der Aufnahme fortwährend weglaufen wollte. Im Gesicht sieht sie nicht mehr so gedunsen aus wie bei der Aufnahme. Schläft nicht mehr so viel wie früher, abends aber doch schon um 7 Uhr. Rechnet eine einfache Aufgabe ziemlich flott aus. Sie meint, daß die anderen Kranken sie auslachen wegen ihrer Dicke.

Nachdem die Kranke weiter 3 Thyreoidintabletten pro Tag bekommen hat und im ganzen $5 \mathrm{~kg}$ abgenommen hat, wird sie am 6. Januar 1909, vielleicht 
etwas weniger gehemmt, entlassen, mit Indikation zur weiteren Thyreoidinbehanlung und mit der Bitte, sie später zur Revision zurückzuschicken.

Da trotz der nach unserem Vorschlag fortgesetzten Behandlung ihr Zustand sich namentlich psychisch allmählich verschlimmerte, wird auf Ersuchen des Arztes die Kranke am 13. April 1910 wieder in die Klinik aufgenommen.

Der Status morbi ist:

Keine Hemianopsie, auch keine bitemporale. Seitwärtsblicken koordiniert, ohne Nystagmus. Beim Augenöffnen bleibt das linke Lid etwas zurück, ist aber leicht geschwollen. Vorübergehend eine Andeutung von rechter Facialisparese ohne Beteiligung der Frontalmuskulatur.

Die Pupillen sind rund, von gleicher Größe, reagieren auf Licht und Konvergenz. Ophthalmoskopisch keine Abweichungen. Fußsohlenreflexe rechts und links plantar.

Beim Gehen, was sie schwerfällig, doch ohne eigentliche Gạngstörung ausführt, steht der rechte Hallux fortwährend in „Babinskischer Stellung“. Ihre Sprache ist langsam, schwertönend. Antwortet richtig auf einzelne einfache Fragen: „Wann sind Sie hierhergekommen ?" „Kennen Sie mich noch ?" Zählt Schlüssel an einem Bund. Hat keine Schmerzen. Läuft zur Türe, will hinaus. Die Haut ist trocken und außerordentlich dick; auf dem Rücken einige blaue Flecken. Auf ihrem Bett liegend, trinkt sie ein Glas Wasser, das Wasser läuft dabei an dem Kinn und an den Kleidern herunter. Das Schlucken macht Schwierigkeiten.

Nachsprechen $555+, 666+$, Spoorwegmaatschappy + , mit leichtem Stimm. tremor. Zählen von $1-20+$ in 18 Sekunden. Von $20-1$ (muß fortwährend angeregt werden ) einmal in 60 Sekunden, nachher in 10 Sekunden. Hierbei leichter Tremor in den Beinen.

Die Untersuchung liefert keine Anhaltspunkte für Aphasie; die speziell auf apraktische Symptome vorgenommene Untersuchung (rechts und links: Hand in die Luft strecken, Zeigefinger in die Höhe strecken, Kaffee mahlen, winken, drohen) fällt ebenfalls negativ aus. Das Drohen begleitet sie mit einem fürchterlichen Gebrüll.

Am 25. April 1910 bekommt die Kranke Temperaturerhöhung unter pneumonischen Erscheinungen. Schwere Somnolenz. Liegt mit geöffnetem Munde und doppelseitiger Ptosis. Bei Berührung der Lippen reflektorisches Schließen des Mundes. Schlucken geht schlecht. Versteht nach wiederholtem Zureden ohne jeden Zweifel. Reagiert nur langsam und mit unvollständig ausgeführten Bewegungen. Von Herderscheinungen ergibt sich nur noch eine deutliche Parese der linken Finger. Fingerbewegungen rechts und Zehenbewegungen rechts und links ungestört. Links außerdem Andeutung von Handklonus. Keine Hypotonie in den Extremitäten.

Das Krankheitsbild bleibt in den nächsten Tagen im großen ganzen das gleiche; beim Versuche zu sprechen macht sie den Eindruck einer schweren bulbären Störung, produziert nur vokale Laute.

Am 20. April 1911 erkennt sie mit der linken Hand ohne nennenswerte Tastbewegungen prompt verschiedene Objekte. Die Fingerbewegungen rechts und die Zehenbewegungen sind ungestört. Die Ptosis ist, namentlich links, etwas zurückgegangen. Die Temperatur neigt wieder zur Norm.

Eine Seitenaufnahme des Schädels zeigt röntgenologisch keine Veränderungen. Wassermann im Blut negativ.

Am 22. April 1910 ist die Kranke inkontinent, klagt über Schmerzen in den Seiten (vom Liegen wahrscheinlich, Decubitus), schluckt schlecht, am besten breiartige Speisen. 
23. April 1910. Temperatur wieder gestiegen. Allgemeiner Zustand wie oben; somnolent. Keine Veränderung der neurologischen Symptome.

Am 23. April 1910 Exitus.

Zusammenfassend haben wir bei der 36 jährigen Frau ein seit ungefähr 31/2 Jahren bestehendes, langsam progressives Leiden anzunehmen, als dessen Hauptsymptom eine apathische Demenz mit schwerer Hemmung in den Vordergrund tritt, körperlich begleitet von einer myxödemähnlichen Zunahme des Körpergewichtes. Nach dem Hinzutreten von etwas verwaschenen und teilweise passageren Herdsymptomen (Facialisparese, Ptosis, schweren bulbären Erscheinungen, links Fingerparese) führte eine komplizierende Pneumonie den Exitus letalis herbei.

Das Bild war uns in mancher Hinsicht unklar. In der ersten Zeit schien die Annahme eines thyreogenen Krankheitszustandes noch am meisten verlockend. Die spätere Aufnahme vergrößerte die Schwierigkeit einer klassischen Umrahmung des Symptomenbildes noch bedeutend: die Herdsymptome forderten weitere differentialdiagnostische Utberlegungen: an Tumor cerebri, speziell der Hypophyse wurde gedacht, die progressive Paralyse wurde in den Kreis unserer Erwägungen hereingezogen, am meisten wahrscheinlich schien aber doch zuletzt die Annahme einer pseudobulbärartigen Krankheit im weitesten Sinne, kombiniert mit oder ev. in engerem Zusammenhang mit thyreogenen Veränderungen.

Die Obduktion am 25. April 1910, 14 Stunden p. m., lieferte außer den erwarteten pneumonischen Erscheinungen und einigen kleinen weißlichen Fleckchen in der Aorta, eine Ùberraschung. Das Gehirn war gleichmäßig stark atrophisch und wog bei einer Schädelkapazität von $1500 \mathrm{ccm}$ nur $1000 \mathrm{~g}$. Ein starker Hydrocephalus externus war vorhanden. Die Gyri wiesen eine erhebliche Verschmälerung auf, die Pia war verdickt und trübe. Bei der nach der Virchowschen Technik gemachten Hirnsektion fand sich von Herden nichts; auch die Obduktion des Rückenmarks lieferte makroskopisch nichts Vermeldenswertes.

Dem Anschein nach hatten wir es also doch mit einer progressiven Paralyse zu tun. Leider hat uns diese - wie sich später erweisen sollte, als zu sicher vermutete - makroskopische anatomische Diagnose veranlaBt, nur die rechte zerschnittene Hemisphaere zu konservieren und einige kleine Stückchen des Rückenmarks. Außer einem Teil des Frontal- und des Occipitallappens, die in Alkohol eingelegt wurden, ward alles aufgehoben in 10 proz. Formol.

Die Rinde wurde untersucht mit folgenden Methoden:

Toluidinblau resp. Thioninfärbung, ev. nach Vorbehandlung mit ResorcinFuchsin: Mehrere Stücke des Frontal- und Occipitalhirns, und (nach Formolfixierung) Gyr. hippocamp.

Bielschowsky: Verschiedene Partien aus Frontal-, Temporal-, Parietal-, Occipitallappen; Gyr. central; Corn. Ammon, Cerebellum.

Fettfärbung mit Scharlach R.: Material wie bei den Bielschowskypräparaten.

Markscheidenfärbung nach Spielme yer: idem. Außerdem wurde an einem chromierten Stück des Frontalhirns und an mit Gliabeize vorbehandelten Stückchen des Occipital-, Zentral-, Frontalhirns und des Cerebellum nach Einbettung die 
Markscheidenfärbung nach Kutschitzk y-Wolters und Spielme yer angewendet. Erwähnen möchte ich hier kurz, daß auch für in der grünen Beize fixierte, eingebettete Präparate die Spielmeyersche Färbung mir wohl die am meisten zuverlässige scheint.

Außerdem wurden - nicht zur Markscheidenfärbung - alkoholfixierte Schnitte des Frontal- und Occipitalhirns ebenfalls nach der Spielmeyerschen Methode gefärbt.

Methylblau-Eosin nach Mann-Alzheimer: Dasselbe Material wie bei den Bielschowskypräparaten, nach Vorbeizung der Stücke in Gliabeize.

Färbung nach Alzheimer mit Ribbertschem Hämatoxylin: idem ohne Cerebellum und Gyr. centr.

Gliafärbung nach Weigert (an Gefrierschnitten): Brauchbare Präparate erhalten von Frontal-, Temporal. und Zentralwindung und Ammonshorn.

Marchi: Ein Stück Frontalhirn.

Die mikroskopische Untersuchung klärte die Verhältnisse nicht in der zu erwartenden Weise auf. Von einer typischen Paralyse war nicht die Rede, und trotz der sorgfältigsten Ausbeutung des vorhandenen Materials ist es uns längere Zeit richt gelungen, dem histologischen Befund eine bestimmte klassische Bedeutung beizumessen.

Von Herden fand sich auch mikroskopisch in keinem der aus verschiedenen Windungsgebieten stammenden Präparate auch nur die geringste Andeutung. Ebenso fehlen Wucherungen der Gefäße; die Endothel- und Adventialzellen verhalten sich durchaus normal, zeigen keine progressiven Kernveränderungen, keine Protoplasmavermehrung; andererseits sind auch keine regressiven Veränderungen nachzuweisen. Auch die Vorfärbung der Toluidinblaupräparate mit ResorcinFuchsin liefert keine anderen Gesichtspunkte. Für die Annahme arteriosklerotischer Veränderungen an den kleineren Hirngefäßen fehlt somit jeder Anhaltspunkt. Auch finden sich nirgends Infiltrate um die Gefäße; Nisslpräparate aus verschiedenen Teilen des Frontalhirns und des Occipitallappens und (formolfixierte) des Gyr, hippocampus habe ich dutzendweise sorgfältig durchgesehen, ohne auch nur an einer Stelle Plasmazellen oder Lymphocyten in der Rinde zu begegnen.

Nirgends findet sich eine Anhäufung von Abbauzellen. Körnchenzellen habe ich in der Rinde überhaupt nicht angetroffen, vereinzelt vorkommende perivasculär gelagerte Zellen mit fettigen Körnchen oder auch größere Fettklumpen finden sich über der ganzen Rinde, am meisten aber treffen wir Fettanhäufungen um die Gefäße des Frontallappens und des Ammonshorns an. Eine das Normale sehr erheblich überschreitende Fettablagerung möchte ich darin aber nicht anerkenren.

Eine auffallende Gefäßvermehrung oder Gefäßknäuelung fehlt, wie schon aus dem Obenerwähnten hervorgeht, ebenfalls. Corpora amylacea finden sich in erheblicher Menge in den peripheren Schichten des Gyr. hippocampus und Ammonshorns und zerstreut im Rückenmark.

Senile Plaques (Drusen) fehlen in allen Rindenarealen vollständig. Weder in den Methylblau-Eosin-Präparaten, noch in den Bielschowskypräparaten von verschiedenen Frontal-, Temporal-, Parietal-, Occipitalwindungen, von Zentralwindung oder Gyr. hippocampus mit Ammonshorn habe ich auch nur eine Druse zu Gesicht bekommen, trotzdem bei dem weiteren Befund begreiflicherweise eifrig danach gefahndet wurde.

Die Pia zeigt namentlich an den Frontalwindungen deutlich progressive Veränderungen: Fibroblastenwucherung und Fasernvermehrung. Was an der frontalen Pia im Vergleich zu der occipitalen aber am meisten auffällt, ist eine Durch- 
setzung mit zerstreut liegenden Lymphocyten in ziemlich erheblichem Maße. Es sind Zellen mit dunklem, kleinem Kern, und - in den Toluidinblaupräparaten mit deutlichem, ziemlich großem, homogen rosafarbenem rundem Zellleib. Einige zeigen eine Ähnlichkeit mit bestimmten makrophagischen Elementen, ohne jemals auch nur annähernd das Äußerliche von Körnchenzellen anzunehmen. Andere neigen in ihrem Aussehen dem Bilde von Plasmazellen; man trifft entweder Zellen mit den Radspeichenkernen, doch rundem, blassem Zellleib, oder Zellen mit kleinem dunklem Kern, welche ein mehr oder weniger metachromatisch gefärbtes Protoplasma besitzen; kurz, U̇bergangsformen zu Plasmazellen sind nicht sehr selten, trotzdem es mir nur in einem Schnitt gelungen ist, zweifellose typische Plasmazellen nachzuweisen; hier habe ich sie sehr spärlich in der Pia angetroffen, höchstens zwei in einem Gesichtsfeld.

Weiteres Vermeldenswertes liefert die Pia nicht; die Rückenmarkspia ist ohne besonderen Befund.

Die Hauptveränderungen in den Rindenpräparaten weisen die Ganglienzellen auf. In allen Regionen der Rinde finden sich Zellen mit der Alzheimerschen spezifischen Veränderung. Am besten gehen wir hierbei aus von den Silberpräparaten. Wir treffen hier Zellformen an, worin die Fibrillen verdickt sind und sich exquisit argentophil zeigen (vgl. Taf.VII, Figg. 1, 2,3). Daß es sich hier um Veränderungen handelt, die mit den Fibrillen in Zusammenhang stehen, braucht wohl nicht besonders betont zu werden. Mit Bielschow s k $\mathrm{y}^{1}$ ) möchte ich aber darauf hinweisen, daß eine einfache chemische Umwandlung der Fibrillen hier kaum annehmbar ist. Es sind girlandenähnliche Verbindungsschlingen sehr deutlich erkennbar, wo normaliter ein derartiger Verlauf von Fibrillen ausgeschlossen ist. Wie die Veränderung aufzufassen ist, wird deutlicher aus den weiteren Zellbildern. Hier sind erstens Formen zu verzeichnen (Figg. 4, 5, 6, 7, 8, 9), wo sich breitere, mehr band- als fadenförmige Schlingen bilden. Ohne weiteres bekommt man aus diesen Bildern den Eindruck, daß sich hier um einzelne Fibrillen Substanzen abgesetzt haben; aus der dichten Masse des dunklen Bandes in Fig. 4 treten normale Fibrillen deutlich hervor: man sieht ein Fibrillenbündel successive mit anderer Substanz sich einhüllen, bis in der dunklen Masse bald jede Struktur verschwunden ist. Es erscheint mir wahrscheinlich, daß die Entstehung der schön abgerundeten Schlingen, wie in den Figg. 5, 6, 7, wohl auf einem Abreißen der mit Ablagerungen besetzten Teile der Fibrillen von dem normalen Gerüst beruht; mit der Annahme einer Plastizität dieses abgelagerten Stoffes kommt man dann zur Erklärung dieser eigentümlichen Zellbilder vollständig aus. Es scheint mir übrigens wahrscheinlich, daß die primär den Fibrillen anhaftenden Stoffe, ebenso wie das Krystall in der Salzlösung, eine Prädilektionsstelle für weitere Ablagerungen darstellen. Denn die Bilder, wie die der Figg. 1-3, sind in meinen Präparaten außerordentlich selten. Hier sind offenbar sehr viele Fibrillen gleichzeitig ergriffen, die Zellen sind dabei zugrunde gegangen, Kerne sind nicht mehr nachzuweisen, auch nicht bei Verschiebung der Mikrometerschraube. In den anderen abgebildeten Zellen ließ sich ein Kern und Protoplasma deutlich nachweisen; auch in den Zellen wie der Figg. 4, 5, 6 fehlt der Kern nicht immer. Die erstere Veränderung stellt also wohl die schwerere dar, wenn auch nicht anzunehmen ist, daß sie als das Endstadium der betreffenden Veränderungen im allgemeinen zu betrachten ist. Denn - und hier komme ich wieder auf das eben Ausgeführte zurück - es scheint mir unzweifelhaft, daß im allgemeinen der Prozeß anders verläuft, daß die Mehrzahl der Fibrillen einer Zelle durchweg nicht befallen wird, doch die weiteren Ablagerungen um den durch primäre Veränderung eines kleinen Teils der Fibrillen. substanz gebildeten Prädilektionspunkt stattfinden, sei es auch gelegentlich

1) Journ. f. Psychol. u. Neurol. 18. 
unter Benutzung der aus den Polen der Masse austretenden Fibrillen als vorgebahnte Richtungslinien des Appositionswachstums. Schon die breiten Bänder der Figg. 4, 5, 6 machen in ihrer Kontinuität und ihrer relativen Kürze ein solches Verhalten wahrscheinlich. Immerhin muß man auch aber solche Formen in den Präparaten suchen. Weitaus am häufigsten - in der Frontalregion sogar gruppenweise, so daß man ganze Gesichtsfelder damit besetzt finden kann, und im Gyr. hippocampus fast ausschließlich - sind die Zellbilder wie in den Figg. 10-15. Hier findet sich in jeder Zelle ein größerer rundlicher oder mehr ovaler, oft wurstförmiger Klumpen. Oft füllt dieser neben dem Kern den Zellraum fast ganz aus. Struktur ist in der Masse nicht zu entdecken, vereinzelt, wie in Fig. 14, ist eine etwas wellige Anordnung von dunkelen und helleren Partien angedeutet. An eine Zusammenballung von in einer leimigen Substanz eingebetteten zahlreichen Fibrillen wäre hierbei a priori ebenfalls zu denken. Hiergegen spricht aber ihr färberisches Verhalten. Denn diese Kugeln sind ebensowenig wie die breiten Schleifen argentophil in dem Maße, wie es die eigentliche Fibrillarsubstanz ist. Die Färbung ist vielmehr mattbräunlich. Nur dort, wo sich ohne jeden Zweifel Fibrillen befinden, in den Ablagerungen, tritt die kohlschwarze Imprägnation auf (Figg. 1, 2, 3, auch 4). Ich glaube, man kann sich das Verhalten am besten so vorstellen, daß die abgelagerte Substanz qua talis nicht argentophil ist, doch an Fibrillen entlang gelagert, vielleicht rein mechanisch, vielleicht auch in dem Sinne einer Beize, deren Färbbarkeit erhöht. Die Kugeln würden also aufzufassen sein als isolierte Anhäufungen einer besonderen chemischen Substanz, mit einem kleinen Fibrillenteilchen als Punkt der primären Ablagerung. Hiermit ist auch sehr gut in Einklang zu bringen, daß diese Zellen sich im übrigen, so weit es den Kern und das Protoplasma betrifft, genau so verhalten, wie die nichtbefallenen Zellen; wenn tatsächlich größere Fibrillenmengen an dem Entstehen der Kugeln beteiligt wären, würde ein solches Verhalten (vgl. die Zellen der Figg. 1, 2, 3, ohne Kern und Protoplasma) doch sehr unwahrscheinlich sein. Bei dem außerordentlich großen Volum vieler dieser Kugeln ist ihre Zusammenballung aus Fibrillen übrigens eo ipso schon unwahrscheinlich. Dazu kommt, daß sich, wie z. B. in Fig. 11, öfters normale Fibrillen bis an die Kugeln verfolgen lassen, über die sie dann offenbar hinziehen $^{1}$ ). Ein Wachstum dieser Gebilde durch konzentrische Apposition scheint mir also wohl sicher als möglich anzanehmen.

Es braucht kaum gesagt zu werden, daß schon die Möglichkeit ihres Entstehens aus retrahierten Fibrillenmassen übrigens notwendig die Annahme einer halbflüssigen Konsistenz des Stoffes voraussetzt. Wenn man wie Bielschowsky (l. c.: ,,daß dieser Stoff im weiteren Verlauf auch die Fibrillen inkrustieren kann, unterliegt keinem Zweifel") eine Inkrustation als das Wesentliche des Prozesses annimmt, sind Retraktionsprozesse, die zu Bildern wie Figg. 7, 10, 11 usw. führen, natürlich eo ipso ausgeschlossen.

Unzweideutig für die Annahme einer sehr plastischen Substanz sprechen namentlich die Nisslbilder. Die Ausbuchtungen und Eindrücke, die die Klumpen von der Anwesenheit von Vakuolen im Zellplasma erleiden, und das konstante Fehlen von unregelmäßigen oder eckigen Konturen, soweit diese nicht durch Vakuolen bedingt sind, fordern die Annahme einer formbaren Konsistenz zwingend; man vergleiche hierzu die Figg. 1-12 der Toluidinblaupräparate und 17-20 der Carbolfuchsin-Methylenblaupräparate. Die deutlich

1) Unter Berücksichtigung der Launenhaftigkeit, die in bezug auf die Fibrillenfärbung in gewissem Maße auch den Gefrierschnitt-Silberpräparaten, namentlich nicht ideell fixierter Stücke, noch anhaftet, möchte ich aus dem Fehlen der normalen Fibrillenfärbung in manchen anderen Zellen keine bestimmten Schlüsse ziehen. 
auch in den Abbildungen erkennbaren Vakuolen scheinen mit der spezifischen Veränderung wohl in irgendeinem Zusammenhang $\mathrm{zu}$ stehen. Bilder wie die Toluidinblaufiguren 17-20 legen es șehr nahe, daß sie, sekundär sich bildend, durch ihre blasigen Formen die abgelagerten Substanzen auseinander treiben. Denn diese Vakuolen trifft man fast ausschlieBlich in Zellen mit den obenbeschriebenen Veränderungen; in nicht spezifisch veränderten Zellen trifft man größere vakuoläre Räume nur sehr ausnahmsweise. Die Vakuolen bestehen offenbar nicht aus Fett; in Scharlachpräparaten konnten wenigstens nirgends diesen Vakuolen an Form und Größe entsprechende Fettmassen nachgewiesen werden. Wahrscheinlich haben wir es also mit einer Art hydropischer Vakuolen zu tun. Nebenbei möchte ich hier bemerken, daß die Vakuolenbildung auch in den Zellen mit der beschriebenen Veränderung nicht sehr häufig ist. Die Formen der Figg. 1-7 der Toluidinblaupräparate sind den der Figg. 8-13 gegenüber sehr in der Minderheit.

Ưber die chemischen Beziehungen des fraglichen abgelagerten Stoffes läßt sich nur sagen, daß es sicher keine fettige Substanz ist, was außer an den gewöhnlichen Präparaten auch in Scharlachpräparaten deutlich ist; Jod gegenüber verhalten sich die Klumpen refraktär, während sie mit basischen Anilinfarbstoffen und mit Hämatoxylinmischungen sich homogen färben, wenn auch nicht durch Intensität der Färbung hervortretend.

Aus dem Ausgeführten geht schon hervor, daß ich die verschiedenen Bilder nicht als verschiedene sukzessiv auftretende Stadien eines selben scharf umschriebenen Prozesses aufzufassen vermag. Es scheint mir nicht notwendig, mit Bielschowsky anzunehmen, daß die Fibrillen sich in den späteren Stadien ,,inkrustieren“ können. Daß die Formen der Silberpräparate 1-3 spätére Stadien der Zellen 4-15 darstellen, wird wohl kaum als möglich zu erachten sein. Vielmehr scheint es verlockend, anzunehmen, daß in den ersten Fällen eine große Fibrillenmenge gleichzeitig, akut, von dem Prozeß getroffen ist, was zu dem völligen Untergang der Zellen führte, bevor sie größere Mengen des Stoffes ablagern konnten. So bleiben die konturierten Fibrillen tot im Gewebe liegen. Dort, wo nur ein kleinerer Teil der Fibrillen dem Prozeß zum Opfer fällt, kann die Ablagerung den größten Teil des Zellkörpers einnehmen, ohne die Vitalität der Zelle schwer zu beeinträchtigen. Denn, wie schon flüchtig oben mitgeteilt worden ist, die Zellen mit Kugeln, wo eine durch Appositionswachstum des Körpers relativ geringe Schädigung der Fibrillen anzunehmen ist, unterscheiden sich in Hinsicht auf Zellkern und Protoplasma nicht von den überhaupt von dem Prozeß verschont gebliebenen Zellen. Frei im Gewebe liegende Kugeln kommen nicht vor.

Zusammenfassend scheint es mir also wahrscheinlich, daß wir das Wesentliche der Veränderung in einer Ablagerung eines plastischen Stoffes, mit Prädilektion der Fibrillen als Ansatzpunkt, zu suchen haben; die weiteren Ablagerungen schlagen mit Vorliebe, aber nur in der lebenden Zelle, auf schon vorhandenen Partikeln nieder, es kommt so in den meisten Fällen zu Bildung konzentrisch entstandener Kugeln; in Zusammenhang mit Benutzung von Fibrillenbündeln als „Wachstumsbahnen" exzentrisch von ihrem Ausgangspunkt entstandene Bänder können durch Retraktion eigentümliche schlangenähnliche Schleifen zustande kommen lassen.

Regionär sind, wie schon erwähnt, Lob. front. und Gyr. hippocamp. am meisten betroffen. Im Occipitallappen ist die Veränderung nur sehr spärlich anzutreffen; nach längerem Suchen findet man hier erst eine so veränderte Zelle.

Die weiteren Zellveränderungen bestehen aus körnigem Zerfall mit Abblassung des Protoplasmas (Toluidinblau Figg. 14-16): Zellen mit normaler Struktur finden sich in der Rinde nicht. Auch in den typisch degenerierten Zellen 
ist das übriggebliebene Protoplasma körnig blaß und sieht an den Zellgrenzen wie abgerissen aus. Sklerotische Ganglienzellen fanden sich nirgends vor.

Die Zellkerne sind sowohl in den typisch veränderten wie in den anders degenerierenden Zellen groß, hell, mit deutlicher Membran umgrenzt, haben ein metachromatisch sich färbendes Kernkörperchen und weisen sehr oft tiefgehende Einfaltungen auf. Der Kern ist überall nach der Peripherie gerückt.

Es scheint mir wahrscheinlich, daß die nicht typischen Veränderungen überwiegend akuter Natur sind. Erstens spricht das Zellbild qua talis, und namentlich das Befallensein aller Rindenzellen, auch der schon typisch veränderten, dafür, zweitens findet diese Auffassung eine Bestätigung in dem klinischen Verlauf der letzten Krankheitstage.

Feine Fettstäubchen und -körnchen, färbbar mit Scharlach, Osmium, CarbolFuchsin, Eisenhämatoxylin, in den Formol-fixierten Präparaten auch mit Thionin sich grünlich tingierend, kommen in den meisten Zellen vor. In den typisch veränderten lagern sie im Protoplasma, oft eng die kugeligen Gebilde umschließend.

Die Architektur der Rinde ist nicht grob geschädigt, nur in der frontalen Rinde gibt es Stellen, wo die Regelmäßigkeit der Schichtung in leichtem Grade gelitten hat und in erhöhtem Grade ist dies im Gyr. hippocampus der Fall. Eine besondere Vorliebe der Lokalisation in bestimmten Schichten habe ich bei der spezifischen Degeneration nicht wahrgenommen, man findet die Veränderung ganz planlos in allen Rindenhöhen.

Die Degeneration, wie sie oben beschrieben worden ist, beschränkt sich auf das Großhirn. Weder im Kleinhirn, noch im Rückenmark ergaben sich neben den auch sonst öfters vorkommenden Abweichungen, wahrscheinlich vorwiegend akuter Natur, Andeutungen der klumpigen Degeneration.

Die weitere Untersuchung der Großhirnrinde liefert als wichtigen Befund einen ziemlich erheblichen diffusen Markscheidenausfall, am deutlichsten erkennbar an dem Ausfall supraradiärer Fasern. Auch die Tangentialfaserung hat ziemlich schwer gelitten, wenn auch streckenweise noch Reste übrig geblieben sind. Fleckenweiser Ausfall kommt in keinem der Präparate vor. Am stärksten ausgesprochen findet sich der Markscheidenausfall in der Frontalregion (Gyr. hippocamp. nicht untersucht!).

Im Rückenmark sind die Pyramidenseitenbahnen sowohl in Marchi- wie in Markscheidenpräparaten deutlich nachweisbar sekundär degeneriert, beiderseits in verschiedenem Grade.

Die Neuroglia verhält sich im allgemeinen ziemlich indifferent. Leichte Verdichtung der Randfaserung und abnormale Bildung feiner Fasern in den oberflächlichen Teilen der Rinde sind die einzigen anzutreffenden Veränderungen; große Spinnenzellen mit mächtigen Ausläufern, dichte Filzmassen, auch von gröberen Fasern sich bildend, kommen nur im Ammonshorn vor.

Abräumprodukte weisen nur vereinzelte kleine Zellen in der Rinde auf. In diesem Sinne ist wohl das Vorkommen farbchemisch sich wie die lipoiden Körner der Ganglienzellen verhaltender, meist etwas gröberer Körnchen aufzufassen. Diesen Zellen kommt bei ihrer geringen Frequenz und zerstreuten Lage in dem Gesamtprozeß wohl keine besondere Bedeutung zu. Neurophagische Erscheinungen der Gliazellen trifft man in den Präparaten ausnahmsweise; bei dem sonst nicht seltenen Vorkommen viel größerer Mengen neurophagischer Zellen als vielleicht sogar ziemlich unwesentlicher Befund bei Hirnrinden verschiedenster Herkunft, ist eine Bedeutung diesem Prozesse in unserem Falle nicht beizumessen. In den tiefsten Rindenschichten ist eine Vermehrung der Gliazellen vorhanden; es sind dies Zellen mit kleinen, fast homogen dunklen Kernen, wie man sie bei amöboiden Zellen öfters antrifft; Merkmale, daß sie mit Abbaufunktionen betätigt sind 
(Körner oder Fett enthalten, ein deutliches Protoplasma besitzen usw.), weisen sie nicht auf. Stäbchenzellen kommen nicht vor.

Aus der Feststellung der oben erwähnten histologischen Befunde ergibt sich, wie mir scheint, nicht ohne weiteres eine Würdigung des Gesamtbildes in ncsologisch eindeutigem Sinne; hier ist tatsächlich Raum für verschiedene Auffassungen; in erster Linie tordert bei der Differenzierung wohl die progressive Paralyse Berücksichtigung, und wenn ich auch persönlich entschieden der Ansicht bin, daß eine Paralyse auszuschließen ist, so läßt sich doch nicht verkennen, daß man der gegenseitigen Auffassung eine auf gute Gründe gestützte Existenzberechtigung zugestehen kann. Man würde hier zum Vergleich sich auf die atypischen, stationären Paralysen Alzheimers zu berufen haben; weisen doch diese Formen Besonderheiten auf, die ihre Einreihung in das histologische Bild der klassischen Paralyse nicht mit der Leichtigkeit gestatten, welche man nach den grundlegenden Arbeiten Nissls und Alzheimers über die feinere histologische Diagnostik der Paralyse längere Zeit allgemein voraussetzte: die minutiöse anatomische Verarbeitung auch anderer Prozesse hat ergeben, daß die scharfe Abgrenzung der Paralyse wieder schwieriger geworden ist, namentlich den Formen von Gefäßlues gegenüber; man bedenke, daß Spielmeyer kürzlich sogar noch hingewiesen hat auf anatomische Ähnlichkeiten zwischen der Paralyse und der multiplen Sklerose.

In unserem Falle ist es, wie mir scheint, in erster Linie die Verwertung des Plasmazellenbefundes, die bei den differentialdiagnostischen Überlegungen zu verschiedenen Auffassungen zu führen imstande ist. Typische Plasmazellen fanden sich, wie erwähnt, zwar in sehr geringer Anzahl, aber unzweifelhaft sicher in der Pia des Frontallappens vor. In der Rinde habe ich sie nicht angetroffen. Trotzdem ist die Möglichkeit zuzugeben, daß, wenn sich in Dutzenden von Schnitten eines Blocks nur einer findet mit vereinzelten Plasmazellen in der Pia, bei Untersuchung von hunderten Schnitten, auch einmal eine verirrte um ein Rindengefäß liegen könnte. Schnitte von stationären Paralysen mit kaum anzuzweifelnder Diagnose, worin man nach minutenlangem Suchen einmal eine isoliert an einer Gefäßwand liegende Plasmazelle begegnet, werden auch manchem anderen schon zu Gesicht gekommen sein. Im Verein mit den anderen anatomischen Veränderungen verliert in unserem Fall der Plasmazellenbefund als Symptom der Paralyse aber an Wahrscheinlichkeit. Die Ganglienzellenveränderung als solche gegen die Paralyse anzuführen wäre natürlich eine Petitio principii bei den Erfahrungen, die wir bis jetzt über das Vorkommen dieser eigentümlichen Zellerkrankung besitzen. Auffallend ist es dagegen wohl, daß von den gewöhnlich bei der Paralyse vorkommenden, scharf charakterisierten chronischen Krankheitszuständen der Nervenzellen keine Form 
vertreten ist. Die relativ guterhaltene Rindenarchitektur, das Fehlen von Wucherungserscheinungen an den Gefäßen, die unbedeutenden Gliaveränderungen, und der gleichmäßige schwere Ausfall supraradiärer und radiärer Fasern bei teilweiser Erhaltung der Tangentialfaserung bilden aber wohl einen Komplex, bei welchem die Diagnose der Paralyse sich nicht aufrecht erhalten läßt. Zum Schlusse kommt dazu noch der negative Ausschlag der im hiesigen pathologischen Institut ausgeführten Wassermannschen Reaktion im Blute.

Alles zusammengenommen scheinen $\mathrm{mir}$ in dem Vorhergehenden schwerwiegende Argumente zur Ablehnung der Paralyse zu liegen.

Es kann demnach wohl keinem Zweifel mehr unterliegen, daß unser Fall mit den von Perusini ${ }^{1}$ ), Alzheimer ${ }^{2}$ ), Sarteschi ${ }^{3}$ ) und Bielschows $\mathrm{k} \mathrm{y}^{4}$ ) nosologisch zusammengehört; ich darf die Kenntnis dieser Fälle wohl als bekannt voraussetzen; es wird dann auch begreiflich sein, daß ich vorläufig unsern Fall als einen atypischen zu betiteln wage, atypisch, insofern manche Züge des Prozesses Abweichungen von dem - vorsichtig zu postulierendem - Typus der bisherigen Beobachtungen aufliefern, atypisch mehr im Sinne einer temporären Arbeitshypothese, die ganz im allgemeinen durch nähere Vergleichung mit den übrigen Fällen zu einer schärferen Formulierung des Wesentlichen der Krankheit eventl. Veranlassung geben könnte. Denn daß ein gewisser Typus sich aus der bisherigen Kasuistik hervorhebt, ist so einleuchtend, daß man bezüglich der präsenilen Fälle mit schwer anders zu deutenden psychotischen und im engeren Sinne neurologisehen Symptomen, mit der spezifischen Zellveränderung und den senilen Plaques als anatomisches Substrat, nur in Hinsicht auf ihre Pathogenese, nicht soweit es ihre grobe Zuweisung zu der fraglichen Krankheit betrifft, noch im unklaren ist. Unsere Kranke war nicht senil oder präsenil, die Krankheit begann etwa im 31. Lebensjahre, und bei der anatomischen Untersuchung fehlten die Drusen.

Es fragt sich, ob wir imstande sind, der Forderung des präsenilen Auftretens auch in unserem Fall einigermaßen entgegenzukommen. Ist zwar der Begriff des Präsenilen an sich ziemlich dehnbar, so daß Perusini sogar meinte, keine Bedenken zu hegen zu brauchen, einen seiner Fälle, einen Mann, bei dem die Krankheit im 40. Lebensjahre schon Symptome machte, unter diesem Gesichtspunkt zu betrachten, bei unserem Fall ginge es ohne jeden $Z_{w}$ weifel zu weit, wenn man hier ohne weitere Dis-

1) Über klinisch und histologisch eigenartige psychische Erkrankungen des späteren Lebensalters. Nissl - Alzheimersche Arbeiten Bd. III.

2) U̇ber eigenartige Krankheitsfälle des späteren Alters. Zeitschr. f. die gesamte Neurol. u. Psychiatr. 4.

3) Rivista sperim. di freniatria $\mathbf{3 5}$.

4) l. c. 
kussion das präsenile Wesen der Krankheit anerkennen wollte. Mit 31 Jahren ist man im landläufigen Sinne nicht präsenil. Man wird also, um unsere Kranke mit den anderen Fällen in dieser Hinsicht von einheitlichem Standpunkt aus zu betrachten, nur versuchen können, in dem Begriffe präsenil nach den Momenten zu fahnden, die außer dem als objektiver Maßstab hervortretenden Alter des Kranken zu dieser Bezeichnung führen: tatsächlich versteht man unter der Feststellung der Präsenilität etwas anderes als die reine Zuweisung der Kranken zu einer bestimmten Altersklasse; so wenig wie man unter dem Klimakterium etwa einen für alle weiblichen Personen gültigen, absolut in der Altersentwicklung festgelegten Zeitabschnitt versteht, ebensowenig gilt dies von der präsenilen Periode. Während aber Etappen, wie Pubertätszeit und Klimakterium sich durch endogen bedingte physiologisch scharf qualifizierte Phasen der normalen Entwicklung kennzeichnen, da fehlt uns für die Fixierung des präsenilen - und natürlich à fortiori des senilen - Wesens ein markanter Mittelpunkt.

Was haben wir unter der Senilität zu verstehen? Die dogmatische Frage, ob das Altern an sich als ein normaler oder pathologischer Prozeß aufzufassen ist, kann natürlich bei unseren Erwägungen außer Betracht bleiben. Ich verweise für derartige allgemeine Betrachtungen auf die Zusammenstellungen von Naunyn in Schwalbes Lehrbuch der Greisenkrankheiten. Von Wichtigkeit erscheint es dagegen, festzustellen, daß pathologische Momente bei dem Zustandekommen der Altersveränderungen eine erhebliche, sei es auch koinzidierende Rolle spielen können; die Arteriosklerose der kleinen Hirngefäße z. B. wird wohl allgemein als ein Faktor anerkannt, der wesentlich die Senilität mit provoziert, und auch bei den Bestrebungen der letzten Zeit, die arteriosklerotischen und einfach senilen Prozesse als streng zu scheidende Formen in der Gehirnpathologie auseinanderzuhalten, kann man nicht umhin, doch wenigstens die Arteriosklerose als eine fast regelmäßige Komplikation des senilen Zustandes zu betrachten. Multiple minimale arteriosklerotische Herdchen und Senilität gehören begrifflich eng zusammen, der Habitus apoplecticus ist der Vorstellung des Präsenilen nicht fremd.

Bevor wir indessen weiterschreiten auf dem Gebiet der Pathogenese, erscheint es wünschenswert, aus den psychischen Erscheinungen des Altwerdens das wesentlich Hervortretende zu eruieren. Die psychische Abstumpfung im weitesten Sinne, Gedächtnısschwäche, Merkfähigkeitsdefekte, Herabsetzung der Urteilsfähigkeit, Abnahme der Tatkraft, der Initiative, verminderte Konzentration der Aufmerksamkeit auf die gewohnten Interessen, Abschwächung der normalen Affekte, kurz ein diffuser A usfall von psychischen Funktionen dürfte wohl - in Utbereinstimmung mit den lehrbuchmäßigen Darstellungen - das Kar- 
dinale, das Primäre in der Summe der mannigfachen geistigen Alterationen des Seniums sein. Die Interesselosigkeit der Kranken und ihr meistens scharf hervortretender Merkfähigkeitsdefekt geben der senilen Verblödung den angeborenen oder anders erworbenen Defektzuständen gegenüber im allgemeinen auch etwas Spezifisches. Andere - psychotische - Symptome, Euphorie, Reizbarkeit, Rededrang, korsakoffähnliche Zustände, immer Anzeichen einer schon schwereren senilen Veränderung, sind sicher nicht ohne weiteres danebenzustellen, sie dürften im Vergleich mit den oben genannten vielleicht mehr indirekter Natur sein. Auch in diesen fortgeschrittenen Graden komplizierter Senilität ist indessen die Verblödung - in weitem Sinne - als. der wesentliche Zug des Zustandes nicht zu verkennen. Anders steht es dagegen mit den leichteren Ansätzen der psychıschen Involution: die Unzulänglichkeit der speziellen Untersuchungstechnik der leichtesten, beginnenden Demenz ist wohl die Ursache des Fehlens einer wohlumschriebenen psychischen Symptomatologie der Präsenilen. Man kann also das allgemeine Bild nur von dem graduell verschiedenen Altersblödsinn deduzieren; es ergibt sich aus dem oben Ausgeführten, daß man auf den Nachweis der Präsenilität im Einzelfall fast immer verzichten muß. Wenn man nun prinzipiell das mehr oder weniger schleichende Auftreten von psychischen Ausfallserscheinungen, namentlich einer gewissen Apathie und Merkfähigkeitsstörung, als essentiell für das präsenile Wesen betrachtet, so finden sich in unserem Fall mit analogen psychischen Erscheinungen, nur in Intensität abweichend, doch Anhaltspunkte für eine Annäherung an die Nosographie der anderen einschlägigen Fälle. Ich weise dabei hin auf den myxödematösen Zustand, der bei keinem der bisherigen Fälle beschrieben worden ist; nur in dem 3. Fall Per u sinis findet sich die kursorische Mitteilung, daß bei der übrigens 65 Jahre alten Kranken ein starkes, gedunsenes Gesicht ... etwas kretinenhaftes Aussehen ... elephantiasisartige Hautverdickung" sich vorfanden. Es kann wohl sicher nicht als Zufall betrachtet werden, daß eine Krankheit ${ }^{1}$ ), die geradezu als prämature Senilität öfters aufgefaßt worden ist - auch die Schilderung des klassischen Bildes in dem Kraepelinschen Lehrbuch deckt sich mit dem geläufigen des Altersschwachsinns in großen Zügen - hier nosologisch die Vertretung der „natürlichen“ Rückbildung übernommen hat. Denn wenn auch der Schilddrüse post mortem leider keine genauere Untersuchung zuteil geworden ist, die klinische Beobachtung im Verein mit der anfänglichen Besserung nach Verabreichung von Thyreoidpräparaten stellt die Wahrscheinlichkeit einer thyreogenen Erkrankung doch sehr in den Vordergrund. Es findet sich somit hier eine auf ganz neuem Wege

1) Vgl. die Ausführungen von Biedl, Innere Sekretion. Wien 1910. S. $73 \mathrm{ff}$. Hier auch Literatur. 
sich hervordrängende Andeutung der inneren Verwandtschaft zwischen interner Sekretion und Alters-,,Degeneration". Auch ohne in die von anderen vertretene weitestgehende Konsequenz zu verfallen, daß die „,normale" Rückbildung einfach eine Folge der Hypofunktion der Thyreoidea sei, kann man doch nicht umhin, in unserem Fall dem Myxödemkomplex für das Zustandekommen der Hirnveränderungen eine analoge Rolle zuzuschreiben wie dem höheren Alter in den anderen Fällen.

Vorläufig läßt sich damit feststellen, daß der Boden für die spezifische Zellveränderung unter diesen beiden Umständen vorhanden ist. Indessen drängt sich sofort die Frage auf, ob sich aus der Annahme obiger Tatsachen nicht möglicherweise nähere Beziehungen zwischen Genese der Rückbildung und dem Zustand unserer Patientin ableiten lassen. Waren die Kausalitätsbeziehungen des Alters zu der Thyreoideafunktion, wie auch Biedl feststellt, ziemlich hypothetischer Natur einerseits die Annahme einer primären Dignität der Thyreoideafunktion beim Zustandekommen der Rückbildung, andererseits, um nur die äußersten Richtungen zu erwähnen, die gleichfalls berechtigte Auffassung der Schilddrüsenatrophie gemeinsam mit den zahlreichen sonstigen Atrophien einfach als Symptom des Alterns -, in unserem Fall haben wir einen zweifellos primären thyreogenen Komplex vor uns, der zu sonst nur auf präsenilem Boden beobachteten Veränderungen führte. Leider hat in der Kasuistik die Thyreoidea zur speziellen Untersuchung keine Veranlassung gegeben. Ein Hinweis darauf, daß die Schilddrüse in der Zukunft eine genauere Beobachtung verlangen dürfte, ist in den obigen Ausführungen somit vielleicht gegeben. Um somehr aber erscheint dies wünschenswert, weil außer der charakteristischen Zelldegeneration sich noch weitere anatomische Befunde aufdeckten, die eine nicht zu verkennende Kongruenz mit denen des senilen Gehirns aufwiesen: die Wichtigkeit dieser Tatsachen, die in dem deskriptiven Teil dieser Arbeit schon aufgefallen sein dürften, scheint mir hinreichend begründet, um nochmals eine kurze Zusammenfassung der betreffenden Veränderungen zu entschuldigen. Das Verhalten der Neuroglia-Randverdichtung und Bildung feiner Fasern, gleichmäßige Atrophie des Gehirns mit diffusem Markscheidenausfall, starke Hypertrophie der Pia mit Einlagerung zerstreuter Lymphocyten, Utberwiegen der Veränderungen im Frontalhirn und im Gyr. hippocampus mit Ammonshorn sind eigentlich so typisch senile Abweichungen, daß es wohl überflüssig ist, in Hinsicht hierauf noch auf die neueste größere Zusammenstellung der senilen Veränderungen in der Arbeit von Simchowicz') zu verweisen.

1) Histologische und histopathologische Arbeiten von Nissl und Alzheimer 4, Heft 2. 
Indessen bin ich mir völlig klar darüber, daß mit der Verwertung eines thyreogenen Zustandes als Äquivalent des senilen die Sache zu einer zwar einfachen, aber keineswegs ganz endgültigen Lösung gekommen ist. In einer Hinsicht stehen glücklicherweise die Schwierigkeiten aus: es erübrigt wohl, nochmals darauf hinzuweisen, daß wir bei der Kranken eine Arteriosklerose für das Zustandekommen der Krankheit auch indirekt nicht mit verantwortlich machen können. Bei der Erörterung der Senilitätsfrage sind wir dieser Komplikation, die an sich schon für viele senile Symptome verantwortlich gemacht worden ist, enthoben.

Andererseits drängt sich indessen gerade die Bedeutung des Fehlens arteriosklerolischer Veränderungen hervor; es ist auffällig, daß die „künstliche Senilität" bei dem psychisch so schwer veränderten Zustand unserer Patientin geradezu halt gemacht hat; ihrem Äußeren nach war die Kranke durchaus nicht greisenhaft. Ob wir darin doch noch einen Fingerzeig für die Rolle der Arteriosklerose beim Zustandekommen dieser letzten Symptome zu erblicken haben [man vergleiche die von Na un yn (l. c.) erwähnten Mitteilungen über jugendliche Personen von exquisit greisenhaftem Typus auf Grund schwerer Arteriosklerose; auch hier ist es übrigens fraglich, ob die Arteriosklerose als primärer Prozeß aufzufassen ist], wage ich nicht $\mathrm{zu}$ entscheiden; die reinen klinischen Fälle von Jugendlichen mit Arteriosklerose und Senilen ohne diese Gefäßveränderung sind nach beiden Seiten zu selten; und wenn sogar anatomisch die Deutung der arteriosklerotischen Veränderungen den rein senilen gegenüber noch nicht lückenlos geklärt ist, wo die Forschung doch von der Anatomie aus, unter den Auspizien Alzheimers c.s. offenbar ihren Ausgang nimmt, da scheint es mir dazu sehr fraglich, ob die a natomische Unterscheidung in der klinischen Differenzierung wohl ihre konsequente Durchführung erwarten kann.

Wie gesagt, stehen wir indessen vor der Tatsache, daß manche senilen Züge in unserem Falle fehlen: das Ergrauen der Haare und weitere Erscheinungen, namentlich derartige, auf welche der beim ersten Blick zu erkennende greisenhafte Aspekt beruht, die körperlichen Rückbildungssymptome, waren nicht dem psychischen Bilde parallel ausgebildet. Man kann also nur von einer partiellen Senilität sprechen, d. h. der Symptomatologie nach. Es ist dabei sehr wohl möglich, daß der gleiche Prozeß auf dem Gebiete innerer Sekretion je nach dem Alter der betroffenen Individuen die Symptomatologie modifiziert; an anderer Stelle ${ }^{\mathbf{1}}$ ) gelegentlich Ausführungen über die Rolle der Hypophyse, wo sich das Thema am aktuellsten präsentiert, habe ich diese Frage

1) Zeitschr. f. Nervenheilk. 41. 
näheren Betrachtungen unterzogen. Es scheint mir hier zu weit zu gehen, einen Versuch zu wagen, bei Ausschaltung der Bedeutung der Arteriosklerose, außer der Feststellung dieser Tatsachen, diese mit einer bestimmten Theorie, etwa der Metschnikoffschen Phagocytenlehre des Alterns, in Einklang zu bringen. Jedenfalls gehört zur kompletten „Fälschung“" eines senilen Zustandes, wenn überhaupt denkbar; mehr als die isolierte Sekretionsanomalie einer Blutdrüse, wie man das bei dem Myxödem in bezug auf die primäre Schilddrüsenveränderung annimmt. Das dunkle Gebiet der inneren Sekretion, namentlich in den Wechselbeziehungen ihrer Komponenten, gestattet uns noch in weiter Ferne nicht, etwa wie ein raffinierter Antiquar mit zahlreichen auf das Abkürzungsprinzip basierten chemischen Kniffen neue Kunstobjekte zu altern versteht, so die Bedingungen für eine künstliche Alterung des lebenden Individuums zu eruieren.

Wir sind hiermit an die Frage der Drusen angelangt, die auch eine Altersveränderung bilden, welche in unserem Falle fehlt. An sich wäre dies, wenn wir für die cerebralpathologische Symptomatologie die Nervenzellveränderung verantwortlich machen und die Drusen nur als eine senile Begleiterscheinung auffassen, nicht unverständlich. Fischer fand bei seinem sehr großen Materiale die Drusen nur jenseits des 50. Lebensjahres, und nach den oben entwickelten Anschauungen können wir ohne Bedenken die Möglichkeit anerkennen, daß in unserer PseudoSenilität dieses Symptom sich nicht mit dargeboten hat: für das, ,Warum“ haben wir indessen auf eine Lösung zu verzichten. Die Alzheimersche Auffassung, die in den Drusen ,nur eine Begleiterscheinung der senilen Involution, nicht die Ursache der senilen Demenz" sieht, ist mit dieser Anschauung sèr gut in Utbereinstimmung zu bringen, auch indem er die Bedeutung der Drusen für das histologische Bild der senilen Demenz, in gleichem Sinne wie den Wert der Plasmazellen für das Bild der Paralyse, ausdrücklich anerkennt. Anders wird jedoch die Rolle der Drusen, wenn wir mit der Möglichkeit rechnen, daß sie die anatomisch nachweisbare Ursache einer bestimmten klinischen Symptomengruppe sind; die sorgfältige Zusammenstellung Fischers ${ }^{1}$ ) fordert ein näheres Eingehen auf diese Frage unbedingt. Fischer kommt zu dem Schluß, daß wir in der Sphärotrichie (= Drusenbildung) nicht nur eine eigenartige und besondere Veränderung des Gehirns vor uns haben, sondern eine Gehirnkrankheit im klinischen und pathologisch-anatomischen Sinne, und daß wir sie als Ursache einer ganz bestimmten, klassifizierbaren und auch klinisch diagnostizierbaren Psychose ansehen müssen. Im Gegensatz zu der einfachen senilen Demenz ist dieses klinische Bild das der presbyophrenen Demenz, Verblödung, komplizjert durch

1) Zeitschr. f. d. ges. Neur. u. Psych. 1910. 
presbyophrene Delirien, korsakoffähnliche Zustände, paranoide Symptome. Es scheint mir recht zweifelhaft, ob $\mathrm{Kraepelin}$ geneigt sein würde, die Fischerschen Fälle seiner Alzheimerschen Krankheit ohne weiteres einzureihen; auf S. 628 seines Lehrbuches weist er ausdrücklich darauf hin, ,,daß das klinische Bild mit der ungemein schweren Verblödung, der tiefgreifenden Sprachstörung, den spastischen Erscheinungen, den Anfällen jedenfalls von der Presbyophrenie, wie sie sonst die rein senilen Rindenveränderungen zu begleiten pflegt, in sehr entschiedener Weise abweicht", und weiter: „Die Vorstellung, die wir uns von den Beziehungen der klinischen Störungen beim Altersblödsinn zu den anatomischen Veränderungen machen können, wird im allgemeinen zwei voneinander verschiedene Vorgänge auseinanderzuhalten haben. Finmal findet offenbar ein Untergang von Nervengewebe statt, das zur Bildung von herdartig sich ansammelnden Abbaustoffen und zugleich zu lipoider Entartung von Nerven- und Gliazellen führt. Dieser Vorgang scheint vorzugsweise klinische Bilder von der Art der Presbyophrenie zu erzeugen, bei denen die körperlichen und psychischen Herderscheinungen hinter der allgemeinen Vernichtung des geistigen $\mathrm{Be}$ sitzstandes wesentlich zurücktreten..." $\mathrm{Zu}$ den letzten dürften wohl die Fischerschen Fälle als zugehörig betrachtet werden. Ich glaube nicht, daß jemand eine klinische Sonderstellung für diese Zustände als urgent erklären würde. Es scheint mir daher, daß man die rätselhaften Fälle von „Alzheimerscher Krankheit“ nicht a priori als synonym mit den Fällen der Fischerschen Gruppe betrachten darf; es gilt, uns vorläufig völlig über die Bedeutung der verschiedenen Befunde klar zu werden, und namentlich, wenn Fischer (l. c. S. 666, 667) die Perusinischen Fälle, gestützt auf das Vorkommen der Drusen, auch über die rein histologische Identifizierung dieser Bildungen hinaus als zugehörig zu seiner „Drusenkrankheit" betrachtet, so ist dabei doch wohl im Auge zu behalten, daß in allen Fällen Perusinis die spezifische Zellveränderung sich vorfand, und das klinische Bild sich nur mit noch weiterer Ausdehnung der an sich schon sehr weit gezogenen Grenzen der ,,presbyophrenen Demenz" hierin unterbringen läßt.

Es scheint vorderhand unmöglich, zu einer einheitlichen Auffassung der verschiedenen Befunde zu gelangen. Trotzdem möchte ich mich zu der Ansicht bekennen, daß man mit der Annahme eines spezifischpsychotischen Zustandes mit Drusen als Ursache sich am besten sehr reserviert verhält. Trotz der Bemühungen Fischers hat die Alzheimersche Auffassung der Bedeutung der Drusen noch die verlockendste Wahrscheinlichkeit für sich. Bei aller Eigenartigkeit der Drusen ist eine gewisse Analogie zu den Corpora amylacea nicht zu verkennen; mir ist nicht bekannt, daß man trotz der Vorliebe des Auftretens dieser Körperchen unter bestimmten Umständen, worunter auch das Senium 
zu rechnen ist, ihnen noch eine andere als histologisch-symptomatologische Bedeutung beimessen wollte. Über histologische Ähnlichkeiten zwischen Drusen und Corpora amylacea hat Bielschowsky l. c. sich in letzter Zeit geäußert.

Daß indessen durch diffuse Infiltration oder Einschließung des Nervengewebes (Fischer, 1. c., S. 392) es ausnahmsweise zu einer schwereren Destruktion des Gewebes kommen kann, ist eine Frage, die, auch wenn wir sie bejahen, streng von jener der Spezifität des klinischen Folgezustandes zu trennen ist.

Nach alledem - ich weise hierbei auch auf die von Alzheimer, 1. c., S. 380, beschriebenen zwei einschlägigen Fälle ohne Drusen hin scheint es mir, daß der Begriff der Alzheimerschen Krankheit mit dem Vorkommen der Drusen nicht fest verwachsen ist.

Ich möchte indessen auch der Alzheimerschen Zellveränderung als solche keine bestimmte Bedeutung in bezug auf ein spezifisches Krankheitsbild beimessen. Die Zellveränderung ist jetzt wiederholt beobachtet worden in Fällen einfacher seniler Demenz, ohne saß sich dabei spontan eine besondere Veranlassung geboten hätte, diesen eine Sonderstellung in dem Rahmen der senilen Demenz einzuräumen. Fischer fand in 17\% seiner Fälle die klumpige Veränderungen der Fibrillen und zieht daraus nur den - von seinem Standpunkt begreiflichen - Schluß, daß sie keine integrierende Veränderung der drusigen Gehirne sind. Wie schon erwähnt, hat auch Alzheimer einen, übrigens aus dem Rahmen der Fischerschen presbyophrenen Demenz fallenden Kranken beschrieben, der, von dem Autor selbst zu den betreffenden Fällen gerechnet, nur Drusen und keine Alzheimersche Zellveränderung darbot. In diesem Fall wichen die Zellveränderungen nicht von dem bei Senilen bekannten Bilde ab. Und sogar Spielmeyer, dessen Referat in der Zeitschr. f. d. ges. Nervenheilk. u. Psych. vom 30. Juni d. J. ich eben zu Gesicht bekomme, kann sich bei seiner Forderung einer ,Aufteilung der senilen Verblödung in natürliche Krankheitseinheiten" offenbar nicht entschließen, der Alzheimerschen Zellveränderung in dieser Beziehung eine besondere Bedeutung zuzuweisen. Vorläufig werden wir also am besten die Alzheimersche Zellveränderung einfach als eine neue Form auffassen, die neben den bekannten von Nissl zusammengefaßten Arten der Ganglienzellenveränderungen eine berechtigte Sonderstellung einnimmt, ohne damit über ihren Wert etwas zu präsumieren. Wenn auch diese Ganglienzellenveränderung im Vergleich zu den Drusen nicht nur hauptsächlich symptomatologischer Natur sein dürfte, so müssen wir doch vorläufig das wesentliche ihres Einflusses mehr in das Befallensein der Ganglienzellen ganz im allgemeinen als in der determinierten Veränderung suchen. Mit spezifischen Ganglienzellveränderungen bei spezifischen 
Zuständen ist die Psychiatrie bisher nicht verwöhnt. Vielleicht könnte für denjenigen, der der neuen Zellveränderung doch noch einen derartigen ev. Wert zuschreiben möchte, indessen unser Fall in heuristischer Hinsicht dabei ein bescheidener Fingerzeig sein.

Ich bin hiermit am Schlusse meiner Ausführungen angelangt. Nach alledem, was bis jetzt über die Alzheimersche Krankheit vorliegt, möchte ich mich noch am ehesten der Ansicht Spielmeyers anschließen, der zu ihr rechnet ,,jene Fälle von seniler Demenz, die von dem gewöhnlichen Typus dadurch abweichen, daß sie vor dem eigentlichen Senium auftreten, und daß sich bei ihnen rasch eine schwere Verblödung entwickelt neben Herderscheinungen asymbolischer und aphasischer Art". Nur möchte ich hierbei auch für weitere Herdsymptome die Möglichkeit des Auftretens offen lassen. Insoweit hat man jedenfalls das Recht, eine Alzheimersche atypische senile Demenz zu postulieren, wenn auch nicht ganz in dem Sinne, wie man in bezug auf die Paralyse von einer Lissauerschen atypischen spricht. Die Hauptsache des Atypischen in der Alzheimerschen Krankheitsgruppe scheint mir eben in dem Nichtvorhandensein eines natürlichen, im engeren Sinne senilen Zustandes zu liegen. Für die atypischen tatsächtlich senilen oder hinreichend präsenilen Fälle, zu deren Kenntnis namentlich die lokalisierten Atrophieen Picks den Weg gewiesen haben, scheint mir die Bezeichnung ,Alzheimersche Krankheit", um eine einigermaßen brauchbare Abgrenzung zu erhalten, nicht wünschenswert. Über die Bedingungen für das Zustandekommen dieser explosiven Alterssymptome, die man wegen ihrer Irregularität und der Schwere ihres Charakters nicht mit der einfachen Bezeichnung prämature Senilität oder Präsenilität rubrizieren kann, hat die Fortsetzung der Casuistik sich noch Rechenschaft zu geben. Auch über die anatomischen Befunde sind die Akten noch nicht geschlossen. Es sind zwar in der klumpigen Zelldegeneration und den Drusen zwei symptomatologisch wichtige und wertvolle Merkmale des senilen Gehirnes fast zu gleicher Zeit ans Tageslicht gebracht; trotz dieser Bereicherung unserer histopathologischen Einsichten ist indessen eine definitive anatomische Grundlage für die pseudosenilen Krankheitsfälle hiermit noch nicht zu schaffen; die Alzheimersche Krankheit ist von der Alzheimerschen Zellveränderung nicht ganz abhängig und noch weniger von den Drusen ${ }^{1}$ ). Die klinische Symptomatologie hat für eine Sonderstellung der Fälle mit Alzheimerscher Zellveränderung noch keine Veranlassung gegeben. Fischer erwähnt seine $17 \%$ derartiger Fälle nur nebenbei. Spielmeyer fand sie in 10 von 44 Fällen reiner senilen Demenz. Wenn sich schließlich doch

1) Auch S pielme yer erwähnt in dem Referate Fälle von nichtkomplizierter seniler Demenz mit Drusen; in der hiesigen Klinik sind solche ebenfalls bei spärlichem Materiale beobachtet worden. 
noch ein gemeinsamer ätiologischer Zug finden ließe für all die Fälle, senil oder nicht senil, wo diese Zellveränderung sich darbietet, eine Möglichkeit, die man bei dem vorliegenden Material nicht vollständig überblicken kann, so wäre zuzugeben, daß damit neue Wege zur Abgrenzung einer Krankheit geöffnet werden könnten, die sich dann zwar nicht ganz decken würde mit dem, was man bis jetzt unter Alzheimerscher Krankheit versteht, nosologisch aber jedenfalls eine bessere Einheit darstellen würde. Es könnte sich z. B. herausstellen, daß das höhere Alter nur eine Disposition darstellt zu einem bestimmten, gewissermaßen heterogenen, zu der Zellveränderung führenden Prozeß. Es lassen sich, wie gesagt, diese Möglichkeiten natürlich jetzt nicht übersehen, ebensowenig wie der Einfluß, den die Kenntnis der einschlägigen Krankheit auf die Zahl der Fälle ohne anatomischen Befund - und dies könnte sich sogar ausstrecken bis über die noch immer in den Lehrbüchern figurierenden Hemiplegien sine Materia und ähnliche Affektionen - haben dürfte, sich jetzt genau abschätzen läßt. Es würde aber sicher nicht wundernehmen, wenn ihre Zahl durch die Arbeiten über das vorliegende Thema wieder erheblich einschrumpft.

Ich möchte weiter nicht die Gelegenheit vorbeigehen lassen, hier auf ein ganz anderes Grenzgebiet der obenbeschriebenen Zustände kurz hinzuweisen. Es wird vielleicht einigermaßen befremden, wenn ich als ein solches die Dementia praecox zu nennen wage. Ein von Pick $^{1}$ ) als ,Fall von primärer progressiver Demenz“ beschriebene 41jährige Kranke, bei der sich in ziemlich schnellem Verlauf ein Krankheitsbild entwickelte, daß eine außerordentlich große Ähnlichkeit in seiner psychischen Symptomatologie mit dem Zustand unserer Patientin zeigt, wird von ihm der Dementia praecox eingereiht. Seine Beweisführung - nach Abweisung anderer bekannter Krankheitsbilder schließt er: „Es bleibt also nur die Möglichkeit übrig, den Fall als primäre einfache Demenz, begründet in primärer Hirnatrophie, zu deuten“" - überzeugt mich indessen nicht davon, daß wir in dem Falle tatsächlich eine Dementia praecox anzuerkennen haben, wenn man diese Bezeichnung nicht rein wörtlich faßt. Ein anatomischer Befund des Falles liegt nicht vor. Vielleicht wird mancher nach Kenntnisnahme dieses Pickschen Falles mit mir die Möglichkeit nicht abweisen, daß dieser sonst nicht befriedigend zu erklärende Zustand in unsere Gruppe hineingehört.

\section{Tafelerklärung.}

Alle Zellen sind gezeichnet bei 840 maliger Vergrößerung (Winkelmikroskop, Kompens. Okul. 4, Apochrom. homogene Immers. Aequiv. Brennweite $2 \mathrm{~mm}$ ), Gasglühlichtbeleuchtung.

1) Prager med. Wochenschr. 1904, Nr. 32. 
Tafel VII.

Formolfixierung. Bielschowsky färbung an Gefrierschnitte nach Pyridinvorbehandlung.

Fig. 1, 2, 4, 12, 13, 15 Temporallappen.

Fig. 3, 5, 6, 7, 9 Gyrus centralis anterior.

Fig. 8, 10, 11, 14 Frontallappen.

Tafel VIII.

Fig. 1-16 Alkohol - Toluidinblaufärbung. Fig. 17-20 Alkohol - Carbolfuchsinmethylenblau.

Fig. 1-3, 5-8, 10-20 Frontallappen.

Fig. 4, 9 Occipitallappen. 